\title{
Making Gameful Work Work: The Gamification of Strategic Human Resource Management
}

\author{
Tobias M. Scholz \\ University of Siegen \\ tobias.scholz@uni-siegen.de
}

\author{
Carolin Uebach \\ University of Siegen \\ caro.uebach@gmail.com
}

\begin{abstract}
The recent pandemic enforced a massive digitalization in the working world and enabled the widespread utilization of gamification in organizations. Still, gamification in Human resource management (HRM) is rarely integrated into the human resources (HR) strategy and is often done on an operational level. We intend to portray the conceptual evolution of gamification in HRM. Through integrating the basic rationale of gamification design, we systematically utilize three resulting evolutionary gamification levels - structure, process, and dynamic capability - to explain how gamification in HR matures from a beginner level to a master level to contribute to the leverage of strategic potentials in HRM. At a meta-level, we will discuss the consequences of gamified HRM in terms of professionalization, hybridization, and captivation, finally concluding with a radical gamification vision for HRM.
\end{abstract}

\section{Introduction}

The working world became more digital in recent years due to the increase in automation and the enforced digitalization through the pandemic and the mandatory work from home restrictions. This technological leap enables an organization to utilize gamification broader and more extensively, especially as the whole work experience can be designed in a digital context. The vision of a virtual organization is now feasible, and, subsequently, gamification could be a game-changer in the way HRM can be utilized and designed.

Deterding [1] discusses the potential impact of gamification in management as a choice between the architectural design following a Taylorist understanding or a humanistic approach enabling a positive and meaningful experience for the employees. He thereby states that this humanistic design may lead to a transformative change in the organization. Interestingly, the paper does not discuss the role of HRM in the implementation of gamification in the organization. However, its core role is to create a beneficial environment for the employees.
Gamification in HRM is still under-researched. On this specific topic Murawski [2] only found 45 papers.

It is undeniable that there is a lure to use gamification in the working context. Be it the potential to motivate, the fit towards the gamer culture, a new approach on development, and, finally, the total control of the employee performance [3]. Furthermore, concepts like playbour [4] also hint that not only work can be fun, but a game can be work. Therefore, it is understandable to propose a strong link between both worlds: gamification and the working world. Moreover, we can observe that gaming is increasingly workified in the context of e-sports [5]. Especially because the current pandemic is triggering the disruption of the working world, gamification may be a quick fix to solve the existing problems. Still, research reveals that, even though the pandemic may be temporary, the changes will have long-term economic and social effects and, therefore, strategic implications [6]. HR will face the challenge to integrate structures and processes that proactively support the changes of the future digitized working world [7]. Hence, it is essential to reassess the traditional HR. For that purpose, gamification can be a significant contribution to make work in this digital world more meaningful or gameful [8], and to bridge working and gaming to make sustainable use of strategic gamification systems.

Throughout this paper, we will systematically evaluate the strategic potential of gamification for HRM. To do so, we will start with focusing on the current gamification discourse and deconstruct the concept of gamification. Thereby we identify the current limits in gamification. Building on this, we will use the basic rationale of gamification design to portray the conceptual evolution of gamification in HRM. Based on that, we will introduce a maturity model of HR-gamification. We argue that gamification needs to level up to unlock the strategic potentials of gamification. Therefore, HR must evolve from an operational beginner level (gamification as a structure), throughout an apprentice level (gamification as a process) towards a strategic master level (gamification as a dynamic capability) of gamified HR. The maturity model draws from the concept of dynamic 
capabilities $[9,10]$. Having extended the resource-based view of the company [11], research on dynamic capabilities emerged over 15 years ago [12]. Teece et al. [13] define dynamic capabilities as an organization's ability to use and reconfigure competencies to deal with environmental changes. An updated definition reads: "Dynamic capabilities can be disaggregated into the capacity (a) to sense and shape opportunities and threats, (b) to seize opportunities, and (c) to maintain competitiveness through enhancing, combining, protecting, and, when necessary, reconfiguring the business enterprise's intangible and tangible assets" [9, p. 1319]. With proceeding through each evolutionary level, the HR-gamification matures to being dynamic capabilities of the organization, which fosters flexibility and strategic change. Following that, we will depict the extent to which the resulting gamification advancements in HRM go beyond current gamification, leading to the professionalization as well as a lock-in. Finally, we propose a vision for a radical gamification.

\section{Deconstruction of gamification}

\subsection{Roots of gamification}

The term gamification first started to become popular in 2010 [14]. Researchers have defined gamification in various ways: "the process of game-thinking and game mechanics to engage users and solve problems" $[15$, p. 14] or "gamification refers to a process of enhancing a service with affordances for gameful experiences in order to support users' overall value creation" [16, p. 19]. The most cited definition reads: "gamification is the use of game design elements in non-game contexts" [14, p. 1]. This raises the critical question of what gives game design elements the unique beneficial impact on the non-gaming context.

Therefore, the discourse starts with whether gamification is the correct term for the change of a system [17]. They criticize gamification as relatively narrow and focusing on the one-sided implementation of game elements, such as points, badges, leaderboards, and rewards [18]. The rationale is to reposition gamification more towards "designing contexts as interventions, informed by game design" [19]. This means that gamification, as such, can be positioned on a spectrum between intensity and impact. Gamification can either be relatively operational and superficial or extensive and initiate a type of strategic change. Within the latter category, gamification can support to harness the strategic potentials of an organization.

\subsection{Gamification in human resources}

However, for the purpose of this paper, it is essential to understand that gamification or the utilization of game design elements is rooted in similar theories as to HRM. Concepts of behaviorism, psychology, or system design are prevalent in both academic fields. Nonetheless, the environment and the tools available are, or more precisely, used to be different. On the one hand, video games - the main inspiration for gamification [20] - are rooted in the digital world, and the working world serves as an analogy. On the other hand, video games are designed for fun, whereas the working world aims for productivity. This has changed in recent years. Work has become increasingly digital, and the notion that work should and is, to a certain extent, supposed to be fun has gained traction. Consequently, gamification can serve as inspiration for this transformation [1].

In her literature review, Murawski [2] analyzed 45 papers on the topic of gamification and derived four fields of application: (1) motivation, engagement, and performance, (2) training and development, (3) recruiting and talent management, and (4) knowledge management. Current research is neglecting the long-term effects and the ethical implications of gamification in the work context. As observed in newer studies on gamification in the HR context, this gap in research is still not addressed. One paper focused on employer branding by utilizing serious games [21], which raises the company's cultural fit in the game and reality. In a similar direction goes the paper by Simons et al. [22] concerning the link between gamers and managers. They researched Civilization players and identified that good players were better in problem-solving and planning than worse players. This is not gamification of HR but instead expanding recruiting to include game experience. Another paper highlights the impact of gamification on job satisfaction [23], which mainly used operational game design elements like leaderboards or badges; however, it is unclear if the authors included a potential crowding-out effect in the analysis. It becomes evident that the gamification of HRM still focuses on an operational level rather than on a strategic level. It is understandable, as utilizing it that way, gamification is a quick fix. Still, this approach limits the potential of HRM and may be even harmful in the long run.

\subsection{Integrating the basic rationale of gamifica- tion design in HR}

As explained within the previous section, research and practice of gamification are taking place on a relatively superficial level, therefore, the current use of gamification has not yet fully exhausted its potential. Beyond this, while bringing a short-term benefit, in the long run, 
the gamification system could exert a harmful influence on individual employees, corporate functions such as HRM, and on the organization. We propose that the process of designing a gamification system affects the very core of HRM. Combining the knowledge of game design with that of HRM will create mutual benefits.

It is, therefore, necessary to systematically reevaluate the use of gamification for HRM. In the following, we will do this by building on the rationale of gamification design, meaning that we use the 'level'-principle of games to mature HR-gamification from operational to strategic. This logic is represented within our maturity model in figure 1 . We will explicate the conceptual levels on a step-by-step basis with a long-term perspective aiming for an extensive and holistic gamification application. We argue that while developing gamification from operational to strategic, the organization matures from a beginner-level of gamification as structures, over the apprentice-level by integrating it as processes, towards the master-level of using gamification as a dynamic capability. The last step is associated with the potential of HR to implement a strategic HR-gamification. While HR evolves from the bottom to the top, the gamification matures towards mastery, where full potentials of gamification can be used within HR.

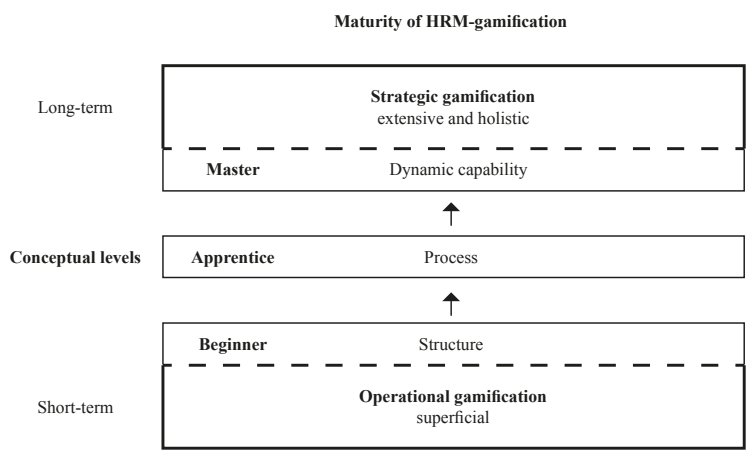

Figure 1: Maturity model of HRM-gamification

With the integration of the basic rationale of gamification, we bridge the gap between the working and the gaming world, and especially merge the phenomenon of gamification with a strategic management research stream. In terms of the dynamic capabilities' analogy, there exists no market for dynamic capabilities, therefore, they cannot be bought, they can only be developed internally [13]. The maturity model of the evolution of the HR-gamification therefore represents the internal development of a sustainable dynamic capability, which first, builds on organizational structures, second develops itself through the interaction of processes, and third matures to being a dynamic capability of sensing, seizing, and reconfiguring [9]. In terms of the video game analogy, such a progressive development could be compared to the learning process in a massive multiplayer online game (e. g. World of Warcraft), in which the player learns the environment (structure), then the skills (processes), and is finally able to utilize all of that efficiently (dynamic capability). It also implies that any gamification system is a dynamic construct that adapts and changes over time. There is no end to the gamification system, as it merges with the HR structure and processes of the organization. As can be seen, with every evolutionary level of gamification, the HRM system becomes more transparent, more individualized, and more strategically agile.

Thereby, the model combines the gamification concept as well as the cycle of dynamic capabilities. Furthermore, this applies as well to the four fields of application by Murawski [2]. (2) and (3) are components of the structure, (1) is linked to processes that facilitate motivation, and (4) can be associated with dynamic capabilities [24]. Therefore, we will use structure, processes, and dynamic capabilities as evolving levels for a gamified HR to progress from an operational to a strategic level. With this, we shift the research of HR-gamification from a short-term based (operational) level to a long-term based (strategic) level.

\section{Leveling HRM up - Using strategic po- tentials}

\subsection{Beginner: Operational gamification as a structure}

The first conceptual level of gamified HR is the beginner level of gamification: the implementation of structural game elements in a non-game environment. It is also the first step for any organization as the people get introduced to gamification, like a tutorial. These structural elements can be points, leaderboards, achievements, levels, clear goals, or rewards [25]. It is also possible to imagine new hierarchical positions such as a "referee" (to be translated as a governance board) or a "contender" (to be translated as an innovation instigator). The adopting system receives new system elements that are to be integrated into the existing organizational and especially HR structures.

Those gaming elements are still primarily operational and imposed onto existing systems. They may improve systems operationally but may also prove harmful in the long run if they either paralyze or overload the system. This short-sighted use of gamification has led to several negatively connoted terms, such as the "electronic whip" [19], "zombification" [26], or "Taylorism 2.0 " [27]. The resulting type of system is highly rooted in behaviorism and reveals the potential of manipulation. The employee receives recognition for a particular 
behavior that top-management deems acceptable. Structuring organizations in this way is comparable to social engineering [28]. Through the structural institutionalization of a top-down perspective, people are nudged [29] into a distinct direction. The effect is reinforced, as this behavior benefits their career in the organization. Anticipatory obedience [30] turns this prediction into a self-fulfilling prophecy [31] or even a self-preventing one [32].

Nevertheless, gamification, even on this relatively simplistic level is bound to impact the organizational structure substantially. Such a reward system, combined with instant feedback, influences, and transforms the entire workflow in an organization. Additionally, this may improve the goal setting, transparency, and the velocity of feedback but may disrupt production and organizational culture. The effect of gamification cannot be restricted to just one isolated organizational segment. Gamification as a structure needs to be considered from a holistic long-term perspective to serve as a foundation for strategic change. Based on that, HR can mature on the path towards mastery.

With the aim of strategically gamifying HRM in a structural term, an adequate infrastructure needs to be implemented. Elements such as scoring points, leaderboards, achievements, levels, reward systems, and player roles can be integrated into HR tasks, for example, training and development or performance evaluation. It is, therefore, essential to define the fit between these game-like structures and the corporate HR structures. Therefore, the organization cannot utilize a standardized gamification system; it is required to analyze the existing HR structure and strategically discuss the adaptation of the gamification elements to the prevailing infrastructure. For this reason, the current organizational signature is just as crucial as the HR-related contextualization of the gaming elements [33]. Especially in terms of employee acceptance [34], an organizational contextualization is of utmost importance. Furthermore, the employee-gamification-fit needs to be assessed prior to implementing a gamification system. Without this fit, gamification systems would only work superficially and interfere with HR, the workforce, and the organization in a detrimental way. Consequently, the ongoing attunement of this fit is essential for the successful implementation of gamification.

Another desirable potential is the real-time feedback [35] provided by a gamification system. It seems reasonable to assume that such synchronicity depends on the input given into the system. Real-time feedback in a game requires intensive work during the design process: the developer anticipates the user's behavior and implements the appropriate feedback loop. Therefore, the game needs to have a wide range of potential responses to the user's actions at its disposal. The same is the case for the gamification system in an organization. The internal environment, however, is unique for every organization. Therefore, the task of HR is to identify and anticipate the potential feedback loops and "patch" the system when the organization evolves and changes. Consequently, as the organization changes, the gamification system needs to change as well especially as the employee is trained, from a behavioral perspective, to respond to the feedback. If the system is not constantly updated and adopted, a diminishing return of creativity and innovational drive in the behavior of the employees may become observable. Without changing the feedback loops, the employees would rely on the existing rules. They would not see any motivation to work outside of the boundaries of the gamification system and the associated real-time feedback. This task cannot be automated through machine learning, algorithms, or big data; however, one essential goal is to motivate employees to be creative, innovative, and think outside the box.

Current developments and the overall trend of datafication [36] play into the hands of HR, especially with the enforced digitization due to COVID-19. The degree to which today's employees can be surveyed already measures up to the information that video game developers collect on their players. The strategic potential to be lifted lies within a transparent usage [37]. Transparency in HRM means that, on the one hand, HR can gain more information about employees since they are stimulated (or even forced) by gamification to reveal more information about themselves. On the other hand, employees gain more information about their corporate data since the overall results of HR activities will likewise be open to being scored and challenged. Transparency, therefore, is not one-directional, and the gamification system can enable employees to analyze the available information as well. At this initial point, essential structures, basic principles, and simple rules are being implemented and become usable. A fit between HR and the gamification system and a gradual convergence of both structures are essential, as this will shape the general tone of a gamified HR.

\subsection{Apprentice: Gamification as a process}

The second conceptual level of gamified HR is the apprentice level. It comprises the application of gamification to business processes (e. g. managerial and organizational processes). Within this intermediate level, gamification is actively integrated into the value creation of HR. Gamification becomes a process feature in the organization and people are actively using the gamification elements in their work processes daily.

Considering the things that have inspired gamification, i.e., game design and, more particularly, video 
game design, reveals the fact that game development requires a high amount of creativity [38]. Aspects such as customization [39], advancement, competition, teamwork, and discovery motivate players in such a way that they stay committed [40] and see progress or growth. Gamification in HR may also utilize the lessons learned from video game design to keep players motivated.

Modern video games are especially suitable from the process perspective because they are designed to keep the player involved. Modern video games that have a long-term orientation (e.g., World of Warcraft) feature a process-centered design as they are games as a service. Although a video game is finite, the video game developers try to transform their game into something infinite, focusing on the ongoing process. Most gamification systems, however, are primarily player-centered [41] or user-centered [42] and lead to a natural ending [43] by finishing the predefined story for the player. The gamification system is finite, but everything beyond this system is neglected [44]. Consequently, the gamification system contains a point at which the employee will ask: What comes next? The gamification system may boost short-term motivation; in the long run, however, this system will harm commitment [40]. Consequently, gamification in the work context should never end.

The design of the learning curve [45] decides on the success of such a "never-ending" gamification system. In massive multiplayer online games, it is of utmost importance that the players stay in the game for an extended period, and the game should neither be too easy nor too difficult. In general, the goal of the learning curve is to "keep raising the bar" [46]. Some games, however, have trouble balancing the difficulty levels. This can lead to frustration and cause players to lose interest in the game. Gamification is currently neglecting the strategic potentials by focusing on closed short-term gamification processes. In particular, to ignore the question regarding the ending is harmful for any organization. What happens, for example, once someone has reached the end of the learning curve? Or, equally harmful, if an employee loses his or her commitment to the organization? Gamification will substitute existing processes in the organization, to which employees have to adjust. But gamification has a natural blind spot concerning the borders of the system's design [44].

In order to gamify HR on the apprentice level in terms of a process, the focus lies on the engagement of the employees. It is vital to engage every employee at an individual level and to make their needs a priority. Gamification can assist HRM in meeting the demands of a customized service provision, for example, by designing individualized learning paths that remain demanding and stimulating. Individual "statistics" serve to support personal development, and character screens provide relevant information about individual key performance indicators, learning progress, skill profiles, and career potentials. The gamification system conveys a sense of mastery [44], a factor that has a substantial impact on motivation and is not purely extrinsic. However, it is also essential for employees to understand the relevance and progress for themselves. The task of HRM is to make the employee part of the narration of their career and link milestones to their professional achievements. Any employee can exhibit the impact on the organization and, at the same time, the progress of its own career path. Nevertheless, it is essential for these paths to co-align to a certain degree. Any imbalance could create a harmful situation. The employee could feel exploited or feel like a freeloader.

In video games, it is normal for players to tinker with [47] and alter their game [48]. Consequently, user interfaces can be instrumentalized for gamification systems [49], for example, to facilitate flow experience [50]. Interfaces can be retrofitted towards the needs of a gamification system, and the structural basis must be implemented. This may be comparable to a sandbox game [51]. The current digitization also revealed early concepts of this in terms of Slack, Discord, or Teams. It is highly probable that, similar to the players of a video game, employees in businesses have the same interest in customizing their user interfaces and adding relevant add-ons.

The above-described potentials of gamification as a process underline two important aspects. First, the integration of gamification within HR processes helps employees to view organizational processes more like open systems which exceed the traditional short-term view. This leverages inter-functional and inter-organizational relationships and perspectives. Second, processes are the connecting elements of the value creation of organizations. Therefore, if HR wants to draw from the potentials of gamification, they need to invest in holistic gamification processes before they can level up to the next evolutionary step of gamified HR. Processes are the necessary criterion for strategic gamification as a dynamic capability.

\subsection{Master: Strategic gamification as a dy- namic capability}

The third conceptual level of gamified HR defines gamification as a dynamic capability. To survive in fastmoving markets, organizations need to invest in the development of their dynamic capabilities. Practical organizational anchors are presented by three dimensions: processes, positions, and paths [13]. Due to previously introducing gamification as a process, it can be depicted as a dynamic capability. This is additionally supported by connecting gamification with the characteristics of 
processes in terms of dynamic capabilities: (i) processes have a static role concerned with internal and external coordination and integration of obtainable resources (e. g. gamification is used for the recruiting-process to 'integrate' new talents into the organization); (ii) processes have dynamic roles like learning (e. g. gamification is used for people development in organizations); (iii) processes have transformational roles (e. g. with gamified development systems, employees are trained for the alertness to sense discontinuities and to reconfigure the organization).

This definition can be linked to gamification, especially as one of the goals of gamification is to foster flexibility [52]. From this perspective, gamification can be understood as a specific dynamic capability of companies which fosters HR to level up to the master level of gamification. It helps companies to flexibly adapt to changing competitive situations and improve the strategy-structure-process-environment-fit by integrating, building, and reconfiguring resources for its market responsiveness process. Gamification systems contribute to the dynamic resource configuration of an organization. However, a gamification system is still designed as a static program [44] embedded in a strict context. Such gamification systems represent a source of irritation, and the top-down approach will lead to resistance [19].

This includes the idea that gamification can be harmful as long as it acts as a static brake [53]. Prevalent in the context of gamification is the usage of dashboards that track customers' progress or, in this case, employees. Employees can customize their profile, collect rewards, and gain experience. This may seem quite dynamic at first glance. However, the underlying system is static and will slow down the organization and make it less dynamic. When implementing a static and superficial gamification system within HR, it will - and can not be used as a dynamic capability. A dynamic capability requires individuality and long-term commitment. Therefore, gamification as a dynamic capability is the master level of gamified HR which is put on a same level as strategic HR-gamification. If HR is able to integrate a holistic gamification system, they reached the top of the maturity model.

For HR to actively design dynamic gamification programs, they can introduce new types of competition among employees and working teams. This would result in a completely new regime of resource allocation, following the competitive idea of gaming. Gamification enforces a market-oriented type of coordination of joint value creation [54], designing HR-related electronic markets where the "games" of information, innovation, motivation, employee development, or resource equipment take place [55]. Like video games, all these elements can be handled within an internal market system of that kind. The transfer prices needed for the coordination can be company-specific currencies, such as overall scores, money, but also the awareness of colleagues as "an excellent player" in terms of "dragon kill points" [56], to be translated to "task accomplishment points." Video games generally contain an internal market, and by maintaining this market, video game designers control their world and enable players to participate in such virtual worlds [57]. The task for HR lies within setting up, regulating, and influencing internal gamified markets [58]. Intervention becomes necessary to retain fairness for both the employees and employers.

Going yet another step further, exploration and exploitation of innovative ideas is crucial in both HRM and games (and is at the heart of what the dynamic capabilities of a firm are supposed to support [59]). Gamification allows to refine the ambidexterity concept of exploration versus exploitation [60]. In games, players are challenged to explore new worlds and new ways. A gamified HR function could utilize the concept of "gameful work" [61]. HR could benefit from gamification by broadening its cognitive base through a gameful creation of HR innovations. Employees are encouraged to explore and exploit, resulting in the feeling of making a contribution. This is similar to the idea of co-entrepreneurship [62] or intrapreneurship [63], which subsequently leads to a sense of co-destiny [64]. They are motivated to playfully co-design and contribute to a playful/gameful organization. In video games, and particularly in virtual worlds, players become the co-authors and co-creators of those worlds [48]. Interestingly, the players know they do not hold any copyrights. Despite that, they actively contribute towards these worlds and gain a certain satisfaction in seeing their ideas come to life in "their" world [48]. Players are also comparable to employees, as they contribute to the organization despite, in most cases, not owning any shares in their organization. Nevertheless, appealing to and intensifying their commitment leads to joint value creation and motivates employees to demonstrate organizational citizenship behavior [65] and voluntarily invest time and energy into the gameful organization.

The dynamic capabilities illustrated above enhance HRM from the perspective of quality of processes and possess the ability to increase the potential of strategic agility. This short-term flexibility plus long-term strategic sustainability, called strategic agility [66], is urgently needed in more dynamic environments [67]. The viability of modern companies is linked to their capability to bridge the paradoxical demands of being flexible and sustainable at the same time. This urges HR to invest in learning gamification [68], thus developing a sense of dynamization. Gamification is a long-term project that will cause tremendous changes. The pivotal aspect is knowledge about gamification, both in HR and 
among employees. Hiring a gamification designer [69] will be a starting point. However, a gamification design should be understood as a collaborative process, and the HR department should take the lead. In addition to this, all employees are required to increase their gamification expertise. The system is too strongly intertwined with the general HR system. Their relationship has evolved into a symbiotic interdependency. It is now essential for the organization's survival to sustain the system of gamification.

\section{Implications}

\subsection{Emerging HR professionalization}

This process of gamification in HR leads to significant changes and an intensive reevaluation of the existing system as well as transformation of the organization. Thereby, gamification catalyzes professionalization [70]: Designing HR structures and processes according to the principles of gamification forces HR experts to anticipate and overcome barriers, which can be considered as "professionalization through the back door."

Furthermore, by understanding gamification as an instrument for lifting strategic potentials, HR can build an integrated gamification system that will adapt to new needs and challenges. HR can automatize most operational tasks and can also be elevated in its strategic potential. Building on this, as gamification matures towards a dynamic capability, HR can contribute to the overall organizational flexibilization and professionalization. By freeing up time and space, HR transforms into a visionary enabler.

\subsection{Gamification lock-in as captivation}

However, besides this professionalization, implementing gamification, also, constitutes a long-term commitment for an organization. The gamification system reaches a path-dependent lock-in stage [71], where the organization is now beyond the point of no return. The reason for this is that a gamified HR cannot be easily removed or replaced. Organizations that start using gamification experience some irreversible transition symptoms. Crowding-out effects [72] will occur, which, especially in terms of motivation, have long-term consequences. Even though people may be intrinsically motivated, their motivation will be replaced by the extrinsic motivation of leaderboards and other gamification elements. Removing the gamification system will lead to less motivated employees. Gamification is, therefore, a strategic commitment of the organization.
This likewise puts employees in a situation comparable to a lock-in. An organization invests in a gamification system. Following the motto "keep raising the bar" [46], employees are always aware of the next step, motivating them in a behavioristic way. This phenomenon mimics the metaphorical carrot on the stick, which is quite popular in game design. People invest considerable time in the system and are always motivated or nudged to invest even more in the game. Reaching one milestone will lead to another. This may feel like a trap, however. The employees invest their career in a particular organization; exiting their organization will take a heavy toll as they lose their investment in their career path. Consequently, employees are committed to the organization, but only out of continuance and for normative reasons rather than out of affection.

\section{Moving beyond: A radical gamification vision for HRM}

After promising the potential of the evolution of a strategic HR-gamification, if it organically matures towards being a dynamic capability, there occurs an intriguing question: What would an ultimately holisticallydesigned, gamified HR, in the terms of a dynamic capability, look like? Contrary to the prevalent type of operational and superficial gamification in HR, a radically visionary gamification solution for HRM with intensive planning, proper design, maturity, and careful coordination could avoid "gamification is bullshit" critics [73] and the reproach of engaging in pure "gamewashing" (in analogy to "greenwashing").

The precondition is the substantial preparation of the initial gamification infrastructure within the organization. This consists of a set of initial rules, essential tools, and interfaces among the employees. Contrary to games where the environment can be designed, the environmental influences can only be steered to a certain extent and are random in real life. HRM is perceived as a designed game - and the game designer does not usually appear in an active role.

Throughout gamified HR, the crucial aspect will be the game experience of the employee (who may work everywhere in the organization, not necessarily in HRM itself). As a game, an employee joining the organization will not know much about the organization, the HR, and his role in co-developing people issues. This is the "spawn point" for an exciting experience journey: The employee starts in a new environment, finds potential options for action, is exposed to all possible risks, and observes all the changes. It is essential that the employee discovers and conquers this new world according to the principle "learn the first steps and understand the game mechanics at a fundamental level." For example, first 
HR tasks can be met, such as negotiating targets and salary agreements with superiors.

The learning curve will constantly rise if the gamified HR is well designed initially, following the maturity model, while progressively challenging the employee. New tasks, as well as new tools, emerge throughout the individual development as the employee starts to define their HRM-related aspirations, for example on training efforts, learns new skills such as judging colleagues as part of peer reviews, and checks out new ways to interact within the organization, for example, in informal networks. The employee collects competencies and capabilities to survive within the organization.

Over time, this leads to a more differentiated understanding of the employee's role within the organization. The employee extrapolates the hidden rules, learning the competitive as well as cooperative regularities. The perspective broadens from the individual to a team-wide focus. The employee realizes that it is essential to cooperate. Teams consist of various experts in different areas. They succeed in the team and further increase individual success by using synergies and opening organizational success. It is reasonable that these teams can resolve even more challenging tasks and gain new knowledge through time. For example, the selection of an expert team can be made based on acquired skills and individual expert scores earned by the previous team contribution peer review results. This calls for transparent development histories on different criteria, accessible to the other employees in the organization. The next level would constitute the cooperative selection (or election) of team leaders and even managers in the organization, the collaborative decision on bonus systems, or the collective distribution of accountability roles.

Finally, the employee will change the game alongside this experience journey, inventing new stories. This way, the employee becomes an HR gamification designer, setting up new infrastructures for the gamified HR: new modes of interaction, new HR-related metrics, and new ways of cooperating in HR issues across the organizational boundaries, even finding new outside players and inviting them to the game. Teams will evolve as temporary teams (pick-up groups) or longterm teams (guilds). HRM stays open world and open end.

\section{Conclusion}

This new form of radically gamified HRM exploits the potential of understanding an organization in a modern way. The company no longer has an HRM - it is an HRM. Every employee and manager are seen as individuals at a granular level while still being an integral part of shaping the people's development and fate in the organization. They directly influence their incentives, create individual learning paths, and experience individualized real-time feedback based on the retrospective personal achievement history. They are linked with long-term predictive simulations of potential future directions. The radically gamified HRM compels the actors to participate while being exposed to the market forces. This demands great expertise, professionalism, and intrapreneurial spirit. Still, overall continuity is a crucial cornerstone of this system, as it moves from an endgame towards a story or episodic approach. There is no finish, and everybody participates in the ongoing storytelling. Finally, the governance for company-wide HR will be spread throughout the organization.

In a system of this kind, employees, and managers sense opportunities to shape the gamified HR system, autonomously seize the opportunities by exploiting it, and proactively reconfigure it to infuse it with life. The HR department is left to act in the background, supervising the organization's people-related engine. Stenros [74] would refer to this as second-order design, which is backed by Salen and Zimmerman: "As a game designer, you can never directly design play. You can only design the rules that give rise to it" [75, p. 168]. Leveraging transparency, individualization, and strategic agility will benefit employees and the organization - mutually making playful dynamics work.

\section{References}

[1] Deterding, S., "Gamification in Management: Between Choice Architecture and Humanistic Design," Journal of Management Inquiry, 28(2), 2019, pp.131-136.

[2] Murawski, L., "Gamification in Human Resource Management-Status Quo and Quo Vadis," German Journal of Human Resource Management, 2020, published online before print.

[3] Scholz, T. M., "Gamification and e-HRM," e-HRM: Digital Approaches, Directions \& Applications, 2018, p. 140.

[4] Taylor, N., K. Bergstrom, J. Jenson, and S. de Castell, „Alienated Playbour: Relations of Production in EVE Online," Games and Culture, 10(4), 2015, pp. 365-388.

[5] Scholz, T. M., Esports is Business. Management in the World of Competitive Gaming, Palgrave Macmillan, Cham, 2019.

[6] Collings, D. G., A. J. Nyberg, P. M. Wright, and J. McMackin, "Leading Through Paradox in a COVID-19 World: Human Resources Comes of Age," Human Resource Management Journal, 2021, published online before print.

[7] Fenech, R., Baguant, P., \& Ivanov, D., "The Changing Role of Human Resource Management in an Era of Digital Transformation," Journal of Management Information and Decision Sciences, 22(2), 2019, 1-10.

[8] Landers, R. N., G. F. Tondello, D. L. Kappen, A. B. Collmus, E. D. Mekler, and L. E. Nacke, "Defining Gameful Experience as a Psychological State Caused by Gameplay: Replacing the Term 'Gamefulness' with 
Three Distinct Constructs," International Journal of Human-Computer Studies, 127, 2019, pp. 81-94.

[9] Teece, D. J., "Explicating Dynamic Capabilities. The Nature and Microfoundations of (Sustainable) Enterprise Performance," Strategic Management Journal, 28(13), 2007, pp. 1319-1350.

[10] Wang, C. L. and P. K. Ahmed, "Dynamic Capabilities. A Review and Research Agenda," International Journal of Management Reviews, 9(1), 2007, pp. 31-51.

[11] Barney, J. B., "Firm Resources and Sustained Competitive Advantage," Journal of Management, 17(1), 1991, pp. 99-120.

[12] Barreto, I., "Dynamic Capabilities: A Review of Past Research and an Agenda for the Future," Journal of Management, 36(1), 2009, pp. 256-280.

[13] Teece, D. J., G. Pisano, and A. Shuen, "Dynamic Capabilities and Strategic Management," Strategic Management Journal, 18(7), 1997, pp. 509-533.

[14] Deterding, S., M. Sicart, L. Nacke, K. O'Hara, and D. Dixon, "Gamification. Using Game-Design Elements in Non-Gaming Contexts," in Proceedings of the Conference on Human-Computer Interaction, Vancouver, Canada, 2011, pp. 2425-2428.

[15] Zichermann, G. and C. Cunningham, Gamification by Design: Implementing Game Mechanics in Web and Mobile Apps, O'Reilly, Sebastopol, CA, 2011.

[16] Huotari, K. and J. Hamari, "Defining Gamification - A Service Marketing Perspective," in Proceedings of the 16th International Academic Mindtrek Conference, Tampere, Finland, 2012.

[17] Deterding, S., "The Ambiguity of Games: Histories and Discourses of a Gameful World," in S. P. Walz, and. Deterding (Editors), The Gameful World: Approaches, Issues, Applications, pp. 24-64, MIT Press, Cambridge, 2014.

[18] Walz, S. P. and S. Deterding, "An Introduction to the Gameful World," in S. P. Walz, and S. Deterding (Editors), The Gameful World: Approaches, Issues, Applications pp. 1-13, MIT Press, Cambridge, 2014.

[19] Deterding, S., "Eudaimonic Design, or: Six Invitations to Rethink Gamification", in M. Fuchs, S. Fizek, P. Ruffino, and N. Schrape (Editors), Rethinking Gamification, pp. 305-331, meson press, Lüneburg, 2014.

[20] Deterding, S., "Gamification: Designing for Motivation," Interactions, 19(4), 2012, pp. 14-17.

[21] Küpper, D. M., K. Klein, and F. Völckner, "Gamifying Employer Branding: An Integrating Framework and Research Propositions for a New HRM Approach in the Digitized Economy," Human Resource Management Review, 31(1), 2021.

[22] Simons, A., I. Wohlgenannt, M. Weinmann, and S. Fleischer, "Good Gamers, Good Managers? A Proof-ofConcept Study with Sid Meier's Civilization," Review of Managerial Science, 15(4), 2021, pp. 957-990.

[23] Silic, M., G. Marzi, A. Caputo and P. M. Bal, "The Effects of a Gamified Human Resource Management System on Job Satisfaction and Engagement," Human Resource Management Journal, 30(2), 2020, pp. 260-277.

[24] Easterby-Smith, M., \& Prieto, I. M., "Dynamic Capabilities and Knowledge Management: An Integrative Role for Learning?" British Journal of Management, 19(3), 2008, pp. 235-249.

[25] Hamari, J., J. Koivisto, and H. Sarsa, "Does Gamification Work - A Literature Review of Empirical Studies on Gamification," in Proceedings of the 47th Hawaii International Conference on Systems Sciences, pp. 1-10, Hawaii, U.S.A, 2014.

[26] Conway, S., "Zombification? Gamification, Motivation, and the User," Journal of Gaming \& Virtual Worlds, 6(2), 2014, pp. 129-141.

[27] DeWinter, J., C. A. Kocurek, and R. Nichols, "Taylorism 2.0: Gamification, Scientific Management and the Capitalist Appropriation of Play," Journal of Gaming \& Virtual Worlds, 6(2), 2014, pp. 109-127.

[28] Peltier, T. R., "Social Engineering: Concepts and Solutions," Information Systems Security, 15(5), 2006, pp. 13-21.

[29] Thaler, R. H. and C. R. Sunstein, Nudge. Improving Decisions about Health, Wealth, and Happiness, Yale University Press, New Haven, 2008.

[30] Lepping, P., "Anticipatory Obedience," The Psychiatrist, 35(7), 2011, p. 275.

[31] Merton, R. K., "The Self-Fulfilling Prophecy," The Antioch Review, 8(2), 1948, pp. 193-210.

[32] Brin, D., "Fiction: Ray Bradbury, an Appreciation," Nature, 486(7404), 2012, p. 471.

[33] Scholz, T. M., "Does Context Matter? Conceptualizing Relational Contextualization" in K. Mitgutsch, S. Huber, J. Wimmer, M. G. Wagner, and H. Rosenstingl (Editors), Context Matters! Exploring and Reframing Games and Play in Context, pp. 89-98, new academic press, Vienna, 2013.

[34] Kavanagh, M. H., \& Ashkanasy, N. M., "The Impact of Leadership and Change Management Strategy on Organizational Culture and Individual Acceptance of Change During a Merger," British Journal of Management, 17(S1), 2006, pp. 81-103.

[35] Whitson, J. R., "Gaming the Quantified Self," Surveillance \& Society, 11(1/2), 2013, pp. 163-176.

[36] Scholz, T. M., Big Data in Organizations and the Role of Human Resource Management. A Complex Systems Theory-Based Conceptualization, Peter Lang, Frankfurt, 2017.

[37] Bennis, W., "Leadership in a Digital World: Embracing Transparency and Adaptive Capacity," MIS Quarterly, 37(2), 2013, pp. 635-636.

[38] Weststar, J., "Understanding Video Game Developers as an Occupational Community," Information, Communication \& Society, 18(10), 2015, pp. 1238-1252.

[39] Turkay, S. and S. Adinolf, "Free to be Me: A Survey Study on Customization with World of Warcraft and City of Heroes/Villains Players," Procedia - Social and Behavioral Sciences, 2(2), 2010, pp. 1840-1845.

[40] Schell, J., The Art of Game Design, 2014, CRC Press, Boca Raton, FL, CRC Press, 2014.

[41] Koster, R., "Player-Centered Design", Ralph Koster's Website, 2005, Available Online: http://www.raphkoster.com/2005/12/07/player-centereddesign, accessed 6-11-2021. 
[42] Norman, D. A. and S. W. Draper, (Editors), User Centered System Design. New Perspectives on Human-Computer Interaction, Lawrence Erlbaum, Hillsdale, NJ, 1986.

[43] Huizinga, J., Homo Ludens. A Study of the Play-Element in Culture, Routledge, London, 1949

[44] Nicholson, S., "A User-Centered Theoretical Framework for Meaningful Gamification," in Proceedings of Games+Learning+Society 8.0, pp. 223-230, Madison, WI, 2012.

[45] Przybylski, A. K., C. S., Rigby, and R. M., Ryan, “A Motivational Model of Video Game Engagement," Review of General Psychology, 14(2), 2010, pp. 154-166.

[46] Scholz, T. M., "What Can We Learn from Game Leadership?" in Proceedings of the IX World Media Economics and Management Conference, Bogotá, Colombia, 2010.

[47] Knorr-Cetina, K. D., The Manufacture of Knowledge: Toward a Constructivist and Contextual Theory of Science, Pergamon, Oxford, 1981

[48] Roncallo-Dow, S., E. Uribe-Jongbloed, K. Barker and T. M. Scholz, "Authorship in Virtual Worlds: Author's Death to Rights Revival?" Journal for Virtual Worlds Research, 6(3), 2013, pp. 1-15.

[49] Johnson, D. and J. Wiles, "Effective Affective User Interface Design in Games," Ergonomics, 46(13-14), 2003, pp. 1332-1345.

[50] Csikszentmihalyi, M., Flow: The Psychology of Happiness, Harper Collins, New York, 1992.

[51] Duncan, S. C., "Minecraft, beyond Construction and Survival," Well Played: A Journal on Video Games, Value and Meaning, 1(1), 2011, pp. 1-22.

[52] Sarangi, S. and S. Shah, "Individuals, Teams and Organizations Score with Gamification: Tool Can Help to Motivate Employees and Boost Performance," Human Resource Management International Digest, 23(4), 2015, pp. 24-27.

[53] Schreyögg, G. and M. Kliesch-Eberl, "How Dynamic Can Organizational Capabilities Be? Towards a DualProcess Model of Capability Dynamization," Strategic Management Journal, 28(9), 2007, pp. 913-933.

[54] Stuart, H. W., "Value Creation: A Coordination Game," Negotiation Journal, 27(2), 2011, pp. 171-190.

[55] Parry, E., "An Examination of E-HRM as a Means to Increase the Value of the HR function," The International Journal of Human Resource Management, 22(5), 2011, pp. 1146-1162.

[56] Malone, K. L., "Dragon Kill Points. The Economics of Power Gamers," Games and Culture, 4(3), 2009, pp. 296316.

[57] Castronova, E., Synthetic Worlds: The Business and Culture of Online Games, University of Chicago Press, Chicago, 2005.

[58] Goggin, J., "Regulating (Virtual) Subjects. Finance, Entertainment and Games," Journal of Cultural Economy, 5(4), 2012, pp. 441-456.
[59] O’Reilly III, C. A., and Tushman, M. L., "Ambidexterity as a Dynamic Capability: Resolving the Innovator's Dilemma," Research in Organizational Behavior, 28, 2008, pp. 185-206.

[60] March, J. G., "Exploration and Exploitation in Organizational Learning," Organization Science, 2(1), 1991, pp. 71-87.

[61] McGonigal, J., Why Games Make Us Better and How They Can Change the World, Penguin, New York, 2011.

[62] Pavlovich, K. and P. D. Corner, "Knowledge Creation through Co-Entrepreneurship," International Journal of Knowledge Management Studies, 1(1-2), 2006, pp. 178197.

[63] Ireland, R. D., C. R. Reutzel, and J. W. Webb, "Entrepreneurship Research in AMJ: What Has Been Published, and What Might the Future Hold?" Academy of Management Journal, 48(4), 2005, pp. 556-564.

[64] Davidow, W. H. and M. S. Malone, The Virtual Corporation: Structuring and Revitalizing the Corporation of the 21st Century, Harper Collins, New York, 1992.

[65] Organ, D. W. "Organizational Citizenship Behavior: It's Construct Clean-Up Time,” Human Performance, 10(2), 1997, pp. 85-97.

[66] Doz, Y. and M. Kosonen, "Embedding Strategic Agility: A Leadership Agenda for Accelerating Business Model Renewal," Long Range Planning, 43(2), 2010, pp. 370382.

[67] Sirmon, D. G., M. A. Hitt, and R. D. Ireland, "Managing Firm Resources in Dynamic Environments to Create Value: Looking Inside the Black Box," Academy of Management Review, 32(1), 2007, pp. 273-292.

[68] Simões, J., R. D. Redondo, and A. F. Vilas, "A Social Gamification Framework for a K-6 Learning Platform," Computers in Human Behavior, 29(2), 2013, pp. 345353.

[69] Raftopoulos, M., "Towards Gamification Transparency: A Conceptual Framework for the Development of Responsible Gamified Enterprise Systems," Journal of Gaming \& Virtual Worlds, 6(2), 2014, pp., 159-178.

[70] Stein, V., "Professionalisierung des Personalmanagements: Selbstverpflichtung als Weg“, Zeitschrift für Management, 5(3), 2010, pp. 201-205.

[71] Sydow, J., G. Schreyögg, and J. Koch, "Organizational Path Dependence: Opening the Black Box," Academy of Management Review, 34(4), 2009, pp. 689-709.

[72] Frey, B. S., "How Intrinsic Motivation is Crowded Out and In," Rationality and Society, 6(3), 1994, pp. 334-352.

[73] Bogost, I., "Why Gamification is Bullshit," in S. P. Walz, and S. Deterding (Editors), The Gameful World: Approaches, Issues, Applications, pp. 65-79, MIT Press, Cambridge, 2014.

[74] Stenros, J., Playfulness, Play, and Games. A Constructionist Ludogoly Approach, Tampere University Press, Tampere, 2015.

[75] Salen, K. and E. Zimmerman, Rules of Play. Game Design Fundamentals, MIT Press, Cambridge, 2004. 